\title{
RF, THERMAL AND STRUCTURAL ANALYSIS OF THE 201.25 MHZ MUON IONIZATION COOLING CAVITY*
}

\author{
S. Virostek, D. Li, Lawrence Berkeley National Laboratory, Berkeley, CA 94720, U.S.A.
}

\begin{abstract}
A finite element analysis has been carried out to characterize the RF, thermal and structural behavior of the prototype $201.25 \mathrm{MHz}$ cavity for a muon ionization cooling channel. A single ANSYS [1] model has been developed to perform all of the calculations in a multistep process. The high-gradient closed-cell cavity is currently being fabricated [2] for the MICE (international Muon Ionization Cooling Experiment) and MUCOOL experiments. The $1200 \mathrm{~mm}$ diameter cavity is constructed of $6 \mathrm{~mm}$ thick copper sheet and incorporates a rounded pillbox-like profile with an open beam iris terminated by $420 \mathrm{~mm}$ diameter, $0.38 \mathrm{~mm}$ thick curved beryllium foils. Tuning is accomplished through elastic deformation of the cavity, and cooling is provided by external water passages. Details of the analysis methodology will be presented including a description of the ANSYS macro that computes the heat loads from the RF solution and applies them directly to the thermal model. The process and results of a calculation to determine the resulting frequency shift due to thermal and structural distortion of the cavity will also be presented.
\end{abstract}

\section{INTRODUCTION}

A previously developed technique [3] using a single ANSYS model to produce electromagnetic, thermal, and structural solutions to RF cavity design problems has been adapted for modeling of the $201.25 \mathrm{MHz}$ cavity (Figure 1). The sequence of analysis steps and the associated results are presented in detail.

The key component in the process is a macro that performs the following steps: 1) reads in the normalized nodal magnetic field results on the surface of the ANSYS RF model, 2) calculates the scaled heat flux at each node based on the total heat dissipation, and 3) applies the heat flux values to a separate surface mesh that is used to map the loads onto the thermal model. The analysis provides the determination of various cavity RF parameters, the resulting cavity wall temperature gradients and stress contours, and the vacuum wall displaced shape for use in frequency sensitivity calculations.

In previous analyses, the technique was applied to cavities with all copper surfaces $[3,4]$. Since the current cavity is copper with beryllium foil windows, the surface resistance is not constant. The macro used to compute the heat fluxes had to be modified to accommodate the use of two materials. To allow rapid analysis of any changes in the cavity geometry during the design process, the finite

*This work was supported by the Office of Science, U.S. Department of Energy under DOE contract number DE-AC03-76SF00098 U.S. element model was generated using parametric input files.

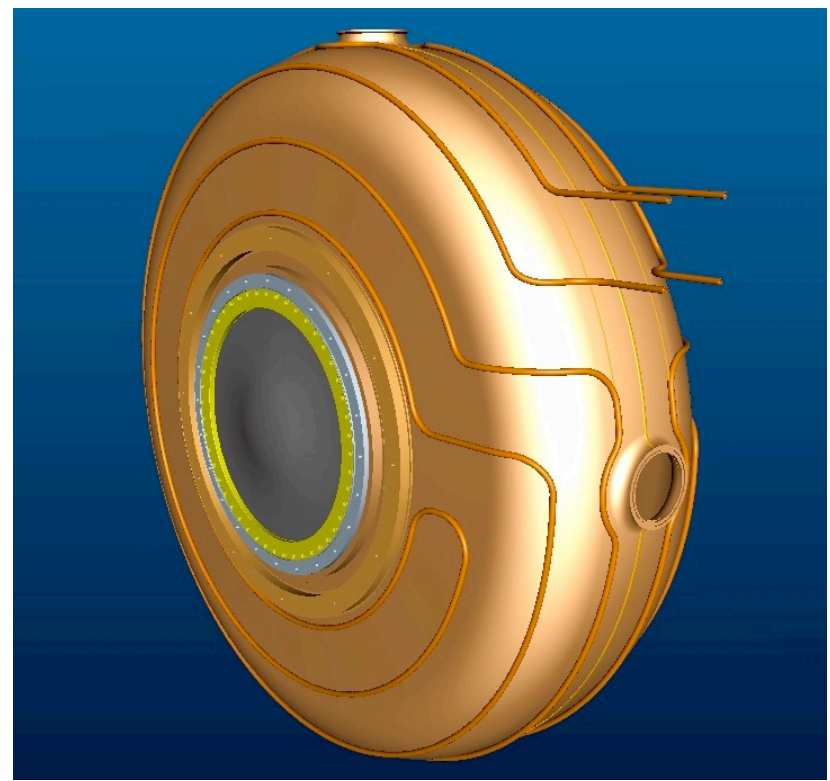

Figure 1: 3D CAD image of the $201 \mathrm{MHz}$ cavity.

\section{RF MODELING}

The initial phase of the analysis consists of a high frequency electromagnetic analysis of the cavity vacuum volume. The model is composed of a solid 3-dimensional volume whose outer surface represents the inner wall of the cavity. In order to reduce the calculation CPU time, the model is constructed to take advantage of symmetry conditions. In the case of the $201 \mathrm{MHz}$ cavity, there are four identical ports for RF input and vacuum pumping located on the cavity periphery and spaced at $90^{\circ}$ apart, resulting in 8-fold symmetry about the beam axis. Note that the cavity ports have not yet been incorporated in the model. The cavity is not symmetric about its mid-plane since one of the beryllium windows curves inward and the other outward.

The resulting volume is meshed with tetrahedral RF elements (HF119) with a finer mesh in areas of expected higher fields and a coarser mesh in the other areas of the cavity. The task of generating a satisfactory mesh is an iterative process. It has previously been shown that electromagnetic results are fairly insensitive to mesh density [3]; however, the surface heat flux is highly dependent on the mesh size at the cavity wall-to-vacuum boundary. By appropriately specifying the local mesh densities, the magnetic fields at the surface and thus the surface heat fluxes are accurately depicted while minimizing CPU time and memory usage. Figure 2 shows the meshed vacuum volume model used to obtain the electromagnetic solution. 


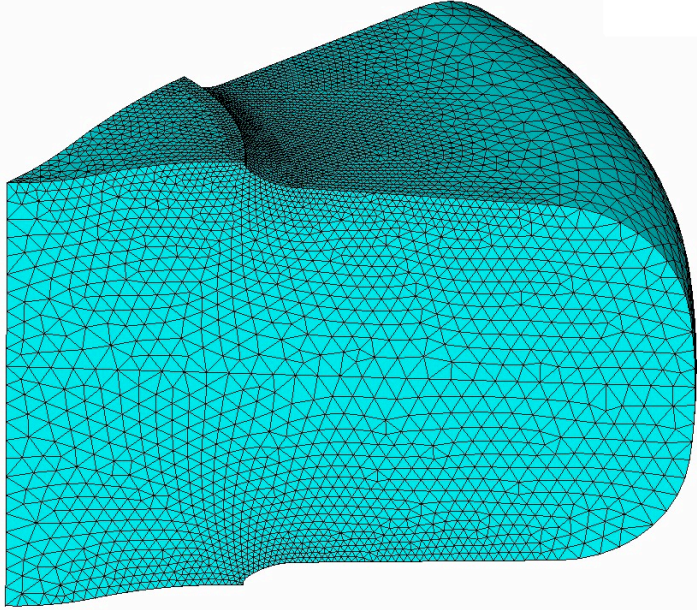

Figure 2: Element plot of the 8-fold symmetric RF model.

Electric wall boundary conditions are applied only to the exterior surfaces of the vacuum volume model that represent the cavity interior wall-to-vacuum interface. In order for ANSYS to compute the $Q$ value for the cavity, impedance boundary conditions are also applied to the same areas in the form of the calculated cavity surface resistances for copper and beryllium. No boundary conditions have to be applied to the model's symmetry planes since these surfaces default to magnetic walls.

The solution to the RF problem is obtained as a modal analysis of the cavity. In addition to the resonant frequency and the $Q$ value $(53,500)$ of the cavity, ANSYS calculates the element results in the form of normalized electric and magnetic field vectors and flux density vectors. This element data is obtained by specifying in the input file that the first mode be extracted. Up to this point, only the cavity geometry and wall material properties have been input to the model; the resulting field data have been normalized within ANSYS and will require scaling before proceeding to the thermal analysis.

\section{THERMAL MODELING}

As part of the physics design and analysis of a cavity, the overall operating parameters and performance characteristics are established. This information includes the peak electric field gradient along the beam axis or the total power dissipated in the cavity walls, which can be used to calculate an appropriate scale factor. In this case, the total power dissipated in the $201 \mathrm{MHz}$ cavity walls $(8.4 \mathrm{~kW})$ has been used to scale the RF results.

A macro written in ANSYS command language is used to read in the normalized total $H$ field solution at each surface node and calculate the associated normalized heat flux based on the surface resistance and the element areas. The heat fluxes are integrated over the cavity wall surface to obtain the total normalized power dissipated in the cavity walls. The difference in electrical conductivity between the copper cavity walls and the beryllium foil windows is taken into account during these calculations. Comparison of the total normalized heat with the expected total power loss in the cavity yields the necessary scale factor. The actual heat fluxes are then calculated and applied to the nodes of a newly generated surface mesh that matches node-for-node the existing mesh on the surface of the RF model. Surface effect elements (SURF152) without mid-side nodes and with heat flux loading capability are used for the surface mesh.

The next step in the analysis requires that the original RF elements be deleted, leaving only the new surface elements with the applied heat fluxes. A solid model that represents the cavity walls and the iris windows is then constructed. The previously described surface elements must be used for the interior cavity wall and window surfaces in order to transfer the heat flux to the thermal model. All features relevant to the thermal performance of the cavity, including the cooling passages, are incorporated in the model (Figure 3). Once the new volume is meshed with the appropriate thermal elements, the heat flux loads are automatically transferred to the model. Convective cooling is applied to the interior surfaces of the water passages in order to achieve heat balance in the model. The resulting temperature contours for $20^{\circ} \mathrm{C}$ cooling water are shown in Figure 4 .

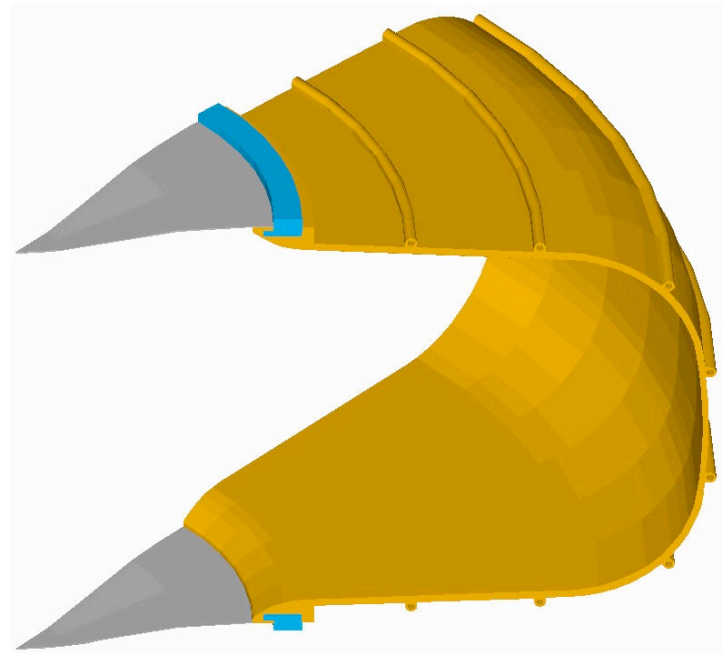

Figure 3: Cavity wall and Be window solid model.

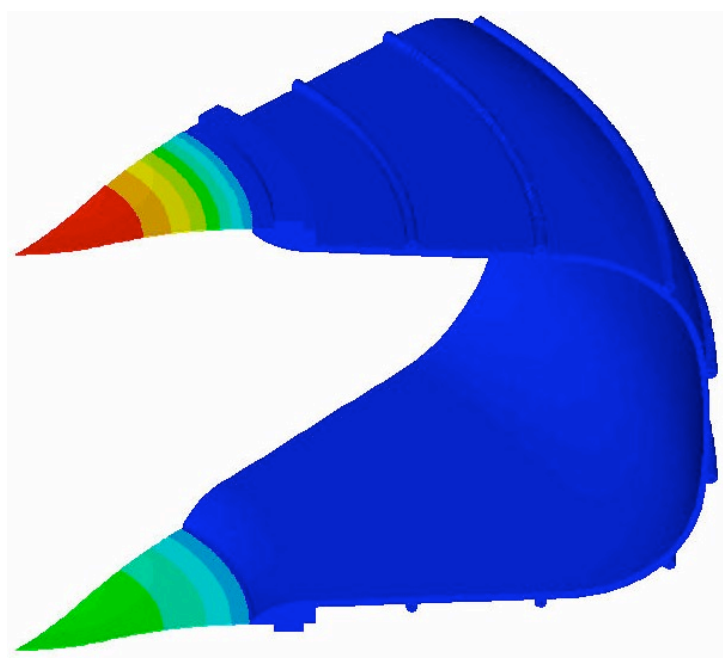

Figure 4: Thermal model with temperature contours. 


\section{STRESS ANALYSIS}

ANSYS allows the thermal elements to be converted directly to structural elements in order to obtain the stress and displacement solutions. The thermal distortion of the cavity is based on the coefficients of thermal expansion of the cavity materials and the nodal temperature data obtained from the thermal solution, which is applied as a load on the structural model. Other loads applied to the model include external atmospheric pressure, symmetry boundary conditions, tuning forces and cavity support constraints. The peak von Mises stress in the $201 \mathrm{MHz}$ cavity body was found to be only $40 \mathrm{Mpa}$.

The cavity's two open beam irises are terminated by $420 \mathrm{~mm}$ diameter, $0.38 \mathrm{~mm}$ thick curved beryllium foil windows. The low $\mathrm{Z}$ beryllium windows are nearly transparent to muons while providing a perfect conducting boundary to RF fields. Since the windows are thin and are cooled only by conduction at their edges, a design was required that would avoid high thermal stresses while also controlling the deflection due to heating. For this reason, a curved window design was developed, resulting in manageable thermal stresses with predictable distortions.

In order to limit the change in cavity frequency as the windows heat up, one window will be installed so that it curves into the cavity while the other curves outward. It was found that the local magnetic fields at the surface of the window that curves inward were significantly higher than on the curving out window. The higher fields result in higher temperatures and stresses as well, although all stresses were found to be below the elastic limit of the beryllium. The stress profiles for the two iris windows are shown in Figure 5.

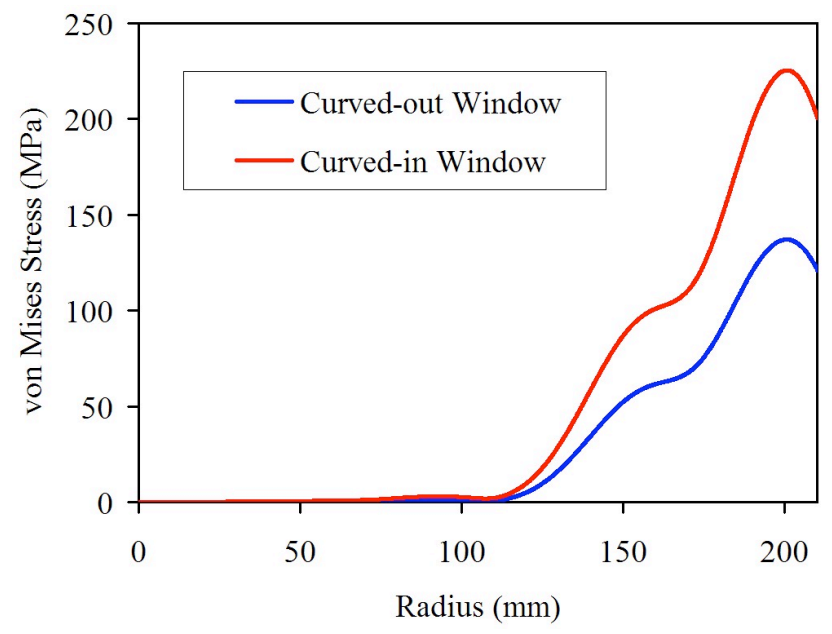

Figure 5: Stress profiles for beryllium windows.

\section{FREQUENCY ANALYSIS}

The structural solution also provides the displacements of the cavity walls due to the various loading conditions. The nodal displacements at the cavity-to-vacuum interface are added to the original nodal locations along a 2dimensional slice of the cavity to yield the resulting displaced shape. A new RF model based on this profile is used to predict changes in cavity frequency due to the various loading and thermal conditions. Since most RF cavities contain non-axisymmetric features such as ports, a similar technique is currently being developed to generate a model of the 3-dimensional displaced cavity shape.

Using the techniques described in the previous paragraph, an analysis has been carried out to determine the frequency shifts associated with cavity distortion due to thermal loading, tuning and external atmospheric pressure. Since the displacements are small and the frequency varies linearly with the load applied, the effects from the various loads can be evaluated separately and the results superimposed as appropriate.

The thermal distortion of the cavity caused a frequency shift of $-94 \mathrm{kHz}$. While some of the shift is caused by the overall expansion of the cavity as the walls heat up, the majority of the shift is due to the fact that the inward curving beryllium window sees more heat flux and deflects more than the outward curving window.

A pair of thick copper rings is welded to the cavity exterior walls to provide an interface for tuning. An external mechanism is used to apply force on the rings, thus distorting the cavity and shifting the frequency. The frequency sensitivity at the tuning ring was found to be $+229 \mathrm{kHz} / \mathrm{mm}$, where a positive displacement is outward from the cavity. The tuning range for the cavity is approximately $\pm 500 \mathrm{kHz}$.

The thin walled copper cavity is not sufficiently strong to support the loads caused by vacuum inside and atmospheric pressure outside. However, the cavity is prevented from collapsing through the support of the tuning mechanism. Since the support is provided only at the location of the tuner rings, some distortion does occur due to the pressure, resulting in a $+54 \mathrm{kHz}$ frequency shift. In practice, the tuning system is designed to cover the range in frequency shifts caused by thermal and pressure distortions.

\section{CONCLUSION}

We have described a multi-step process to characterize the RF, thermal and structural behavior of the prototype 201.25 MHz cavity for a muon ionization cooling channel using a single ANSYS model. The results of the analysis have been used to validate the design and characterize the operating performance of the cavity. This work was supported in part by the US Neutrino Factory and Muon Collider Collaboration.

\section{REFERENCES}

[1] ANSYS is a registered trademark of SAS IP, Inc.

[2] N. Hartman and R.A. Rimmer, "Electromagnetic, Thermal, and Structural Analysis of RF Cavities using ANSYS", PAC 2001.

[3] J.W. Staples and S.P. Virostek, "Engineering Design of the LUX Photoinjector", EPAC 2004.

[4] R.A. Rimmer, et. al., "Fabrication of the Prototype 201.25 MHz Cavity for a Muon Ionization Cooling Experiment", PAC 2005. 